\title{
Does short-term cessation of smoking before lung resections reduce the risk of complications?
}

\author{
Tomoyoshi Takenaka ${ }^{1,2}$, Fumihiro Shoji ${ }^{3}$, Tetsuzo Tagawa ${ }^{1}$, Fumihiko Kinoshita ${ }^{1}$, Naoki Haratake ${ }^{1}$, \\ Makoto Edagawa ${ }^{2}$, Koji Yamazaki ${ }^{3}$, Mitsuhiro Takenoyama ${ }^{4}$, Sadanori Takeo ${ }^{3}$, Masaki Mori ${ }^{1}$ \\ ${ }^{1}$ Department of Surgery and Science, Graduate School of Medical Sciences, Kyushu University, Fukuoka, Japan; ${ }^{2}$ Department of Surgery, Hiroshima \\ Red Cross Hospital and Atomic Bomb Survivors Hospital, Hiroshima, Japan; ${ }^{3}$ Department of Thoracic Surgery, Clinical Research Institute, National \\ Hospital Organization, Kyushu Medical Center, Fukuoka, Japan; ${ }^{4}$ Department of Thoracic Oncology, National Kyushu Cancer Center, Fukuoka, \\ Japan \\ Contributions: (I) Conception and design: T Takenaka; (II) Administrative support: T Tagawa, T Takeo, T Takenoyama, M Mori; (III) Provision of \\ study materials or patients: F Shoji, K Yamazaki, M Takenoyama; (IV) Collection and assembly of data: T Takenaka, F Kinoshita, N Haratake; (V) \\ Data analysis and interpretation: T Takenaka; (VI) Manuscript writing: All authors; (VII) Final approval of manuscript: All authors. \\ Correspondence to: Tomoyoshi Takenaka, MD, PhD. Department of Surgery and Science, Graduate School of Medical Sciences, Kyushu University, \\ 3-1-1 Maidashi, Higashi-ku, Fukuoka 812-8582, Japan. Email: ttake@surg2.med.kyushu-u.ac.jp.
}

Background: Smoking cessation is a highly important preparation before thoracic surgery. We examined the effects of short-term smoking cessation intervention before pulmonary resection on postoperative pulmonary complications (PPCs).

Methods: A retrospective analysis of prospectively collected data was performed for 753 patients who underwent curative surgical resection for thoracic malignancy at 3 institutions. Patients with a smoking history were instructed to quit smoking. After confirming smoking cessation by at least four weeks before surgery, surgical resection was performed. Subjects were classified into three groups based on their smoking status: abstainers (anyone who had stopped smoking for at least 4 weeks but less than 2 months), former smokers (anyone who had abstained from smoking for more than two months prior to surgery), and never smokers (those who had never smoked). We examined the relationship between the preoperative smoking status and PPCs.

Results: Surgery was performed for 660 primary lung cancers and 93 metastatic lung tumors. The smoking statuses were classified as follows: abstainers ( $n=105,14 \%)$, former smokers $(n=361 ; 48 \%)$ and never smokers $(\mathrm{n}=287,38 \%)$. The incidence of PPCs among abstainers, former smokers and never smokers was $15 \%, 8 \%$ and $6 \%$, respectively $(\mathrm{P}=0.01)$. The mean duration of post-operative chest tube drainage among abstainers, former smokers and never smokers was 3.2, 2.2 and 2.2 days, respectively $(\mathrm{P}=0.04)$. The mean post-operative hospital stay among abstainers, former smokers and never smokers was 12.1, 10.6 and 10.2 days, respectively $(\mathrm{P}=0.07)$. There was no 30-day mortality in the cohort.

Conclusions: Short-term smoking cessation intervention did not enough reduce the PPCs as much as in former or never smokers.

Keywords: Lung resection; cigarette smoking; smoking cessation; postoperative pulmonary complications (PPCs)

Submitted Jul 31, 2020. Accepted for publication Oct 15, 2020.

doi: $10.21037 /$ jtd-20-2574

View this article at: http://dx.doi.org/10.21037/jtd-20-2574

(C) Journal of Thoracic Disease. All rights reserved. 


\section{Introduction}

According to Centers for Disease Control reports, as of 2018, approximately $20 \%$ of United States adults currently used any tobacco product, and cigarette smoking reached an all-time low (13.7\%) (1). In Japan, although there is a declining trend in smoking, $19.3 \%$ of the population admitted to being current smokers in 2015 (30\% of men and $8 \%$ of women) (2).

Smoking has many effects on circulation and the pulmonary function (3). It leads to many diseases, including lung cancer, chronic obstructive pulmonary disease, cardiovascular disease and other forms of cancer (3-6). The prognosis of patients with lung cancer depends on the smoking status $(7,8)$. Smoking cessation after a diagnosis of early-stage lung cancer improves prognostic outcomes (7). Smoking is also associated with a high risk of postoperative complications (9). Previous studies indicated that current smokers were twice as likely to experience postoperative pulmonary complications (PPCs) after lung resection in comparison to never smokers or those who had not smoked for more than 4 weeks (9). The decrease in PPCs due to smoking cessation is thought to be related to physiological improvement in ciliary action, macrophage activity, and the small airway function, as well as a decrease in sputum production. On the other hand, several reports have indicated the negative impact of short-term cessation on operative mortality and morbidity (10-12). Mason et al. reviewed the Thoracic Surgery Database, which included 7990 patients, and reported the outcome of smoking cessation on resection of primary lung cancer (11). They found that the risk of PPCs and the risk of hospital deaths decreased with longer intervals of smoking cessation. However, no optimal interval of smoking cessation could be found from the study (11).

Enhanced Recovery After Surgery (ERAS) Society and the European Society of Thoracic Surgeons (ESTS) report that smoking is associated with an increased risk of postoperative morbidity (especially pulmonary complications) and mortality and that ideally, it should be stopped at least 4 weeks before surgery (13). However, the effect of smoking cessation from at least 4 weeks before surgery has been unclear(13).

In this study, we conducted a multicenter retrospective study to evaluate the effects of short-term smoking cessation (at least 4 weeks) on PPCs of patients undergoing thoracic surgery. We present the following article in accordance with the STROBE reporting checklist (available at http://dx.doi. org/10.21037/jtd-20-2574).

\section{Methods}

\section{Patients}

The study conformed to the provisions of the Declaration of Helsinki (as revised in 2013). This study was approved by the institutional review of board of the Hiroshima Red Cross Hospital and Atomic Bomb Survivors Hospital (IRB number: H30-672). Written informed consent was obtained from each patient to use their medical records. We enrolled 753 patients who underwent curative surgical resection for thoracic malignancies between January 2017 and December 2018 from 3 institutions (Kyushu Medical Center, Kyushu Cancer Center and Hiroshima Red Cross Hospital and Atomic Bomb Survivors Hospital) and retrospectively analyzed.

The smoking status was investigated based on selfreporting. Surgeons interviewed the patients about the duration of smoking, number of cigarettes smoked per day and duration of smoking cessation reported the findings from medical record. We instructed active smokers to quit smoking by at least 4 weeks prior to surgery in order to reduce the incidence of pulmonary events in accordance with the ERAS and ESTS guidelines. The patients who could not quit smoking by themselves were offered access to smoking cessation clinics, smoking cessation programs and stop smoking medications. Confirmation of smoking cessation was made by the patient's self-report or family report. Abstainers were defined as anyone who had stopped smoking at least 4 weeks but less than 2 months before surgery, and former smokers were defined as those who had abstained from smoking for more than 2 months prior to the operation. Never smokers were defined as those who had never smoked. In the case of patients who could not stop smoking before surgery, surgery was postponed until they managed to discontinue smoking for 4 weeks.

\section{Data collection and extraction}

Demographic, clinical and treatment data were extracted from an institutional database that included all patients who had undergone thoracic surgery. Operative mortality was defined as death within 30 and 90 days of resection. The PPCs were defined according to the Japan Clinical Oncology Group postoperative complications criteria; grade $\geq 3$ complications were extracted (14). Postoperative 
Table 1 The clinical characteristics of the patients

\begin{tabular}{lc}
\hline Variable & Outcome, $\mathrm{n}=753$ \\
\hline Mean age (range) & $68(18$ to 92$)$ \\
Sex & \\
Male & $426(57 \%)$ \\
Female & $327(43 \%)$ \\
Smoking status & \\
Never smoker & $287(38 \%)$ \\
Former smoker & $361(48 \%)$ \\
Abstainer & $105(14 \%)$ \\
Mean pack year smoking (range) & $28(0-260)$ \\
Cause of surgery & \\
Primary lung cancer & $660(87 \%)$ \\
Metastatic lung tumor & $93(13 \%)$ \\
Surgical procedure & $11(1 \%)$ \\
Partial resection & $167(22 \%)$ \\
Segmentectomy & $33(4 \%)$ \\
Lobectomy & $542(72 \%)$ \\
Pneumonectomy & \\
\hline
\end{tabular}

complications included pneumonia, prolonged air leakage that required drainage for more than 7 days, bronchopleural fistula, empyema, atelectasis detected by chest roentgenography, pulmonary embolism and acute exacerbations of idiopathic interstitial pneumonia. An acute exacerbation of interstitial pneumonia was defined as worsening of dyspnea and hypoxia from baseline within 30 days, including new ground-glass abnormalities or consolidation on a background of a reticular or honeycomb pattern.

\section{Statistical analysis}

Differences between the groups were tested for significance using the Kruskal-Wallis test for continuous variables and Pearson's Chi-squared test for nominal variables. Univariate and multivariate logistic regression analyses were performed to identify predictors of PPCs. Variables that were significantly associated with PPCs in a univariate analysis were included in a multivariate analysis using a Cox proportional hazards regression model. The analysis was performed using the JMP software package (version 11, SAS
Institute Inc.). $\mathrm{P}$ values of $<0.05$ were considered to indicate statistical significance.

\section{Results}

The characteristics of the 753 patients are summarized in Table 1. The patients were categorized, based on their smoking history as follows: abstainers $(\mathrm{n}=105,14 \%)$, former smokers $(\mathrm{n}=361 ; 48 \%)$ and never smokers $(\mathrm{n}=287$, $38 \%$ ). All patients were diagnosed with primary lung cancer or metastatic lung tumor and received standard curative resection. Surgery was performed for 660 primary lung cancers and 93 metastatic lung tumors. The types of procedures performed included lobectomy $(n=542)$, pneumonectomy $(n=11)$, wedge resection $(n=167)$ and segmentectomy $(n=33)$. There were significant differences among the three smoking status groups with regard to sex, vital capacity (VC), \% VC, forced expiratory volume in 1 second (FEV1.0), FEV1.0\% and \%FEV1.0 (Table 2). The abstainers were significantly more likely to be men and to have lower \% VC and FEV1.0\% values (Table 2).

PPCs occurred in 62 of the patients $(8.2 \%)$ in the overall population. In comparison to never smokers and past smokers, abstainers had a higher frequency of PPCs $(6 \%, 8 \%$ and $15 \%$, respectively; $\mathrm{P}=0.018)$ in the overall population (Table 3). There was no significant difference in the frequency of PPCs between the never smoker and past smoker groups. Among patients with primary lung cancer, PPCs were observed 15 patients (16\%) in the abstainer group, 28 patients $(8 \%)$ in the past smoker group and 16 patients $(6 \%)$ in the never smoker group $(\mathrm{P}=0.019)$. In the overall population, the mean duration of post-operative chest tube drainage among abstainers, former smokers and never smokers was 3.2, 2.2 and 2.2 days, respectively $(\mathrm{P}=0.038)$. The mean post-operative hospital stay among abstainers, former and never smokers was 12.1, 10.6 and 10.2 days, respectively $(\mathrm{P}=0.073)$. There was no 30 -day mortality.

The distribution of PPCs is shown in Table 4. The most common PPC was prolonged air leakage and the second most common PPC was pneumonia. Exacerbations of Interstitial pneumonia occurred in the past smoker and abstainer groups, but not in the never smoker group.

A univariate analysis determined that sex, smoking status, pack year smoking, surgical procedure and type of diseases were factors that influenced the occurrence of PPCs in the overall study population. Furthermore, a multivariate analysis revealed that sex and the surgical procedure were 
Table 2 Characteristics of the subjects categorized according to smoking status

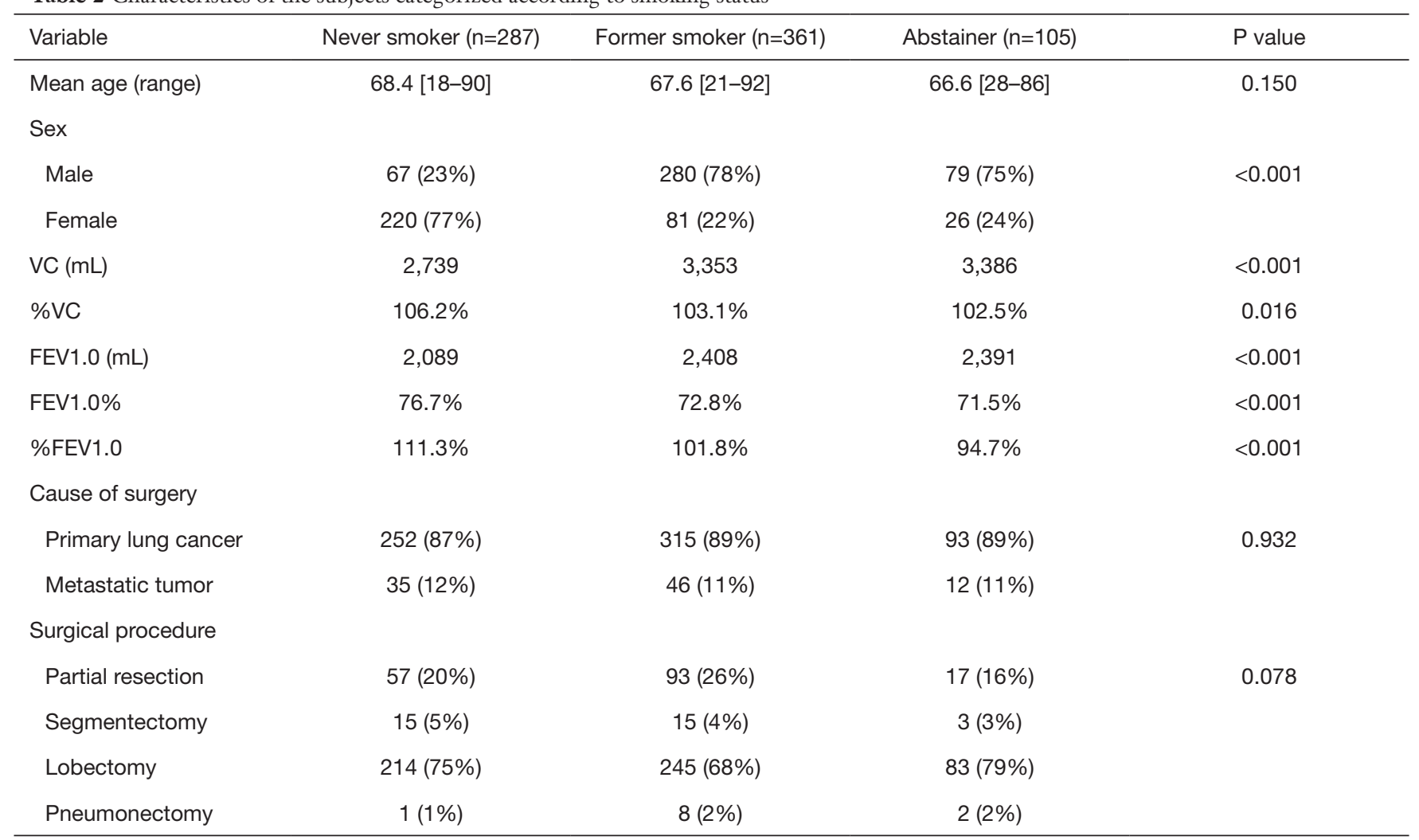

VC, vital capacity; FEV1.0, forced expiratory volume in 1 second.

Table 3 Short-term outcomes in patients stratified by smoking status

\begin{tabular}{|c|c|c|c|c|}
\hline Variable & Never smoker $(\mathrm{n}=287)$ & Former smoker $(n=361)$ & Abstainer $(n=105)$ & $P$ value \\
\hline \multicolumn{5}{|l|}{ PPCs } \\
\hline No & $270(94 \%)$ & $332(92 \%)$ & $89(85 \%)$ & 0.018 \\
\hline Yes & $17(6 \%)$ & $29(8 \%)$ & $16(15 \%)$ & \\
\hline Mean tube drainage (day) & $2.2[1-23]$ & $2.2[1-41]$ & $3.2[1-15]$ & 0.039 \\
\hline Mortality & 0 & 0 & 0 & \\
\hline
\end{tabular}

PPCs, postoperative pulmonary complications.

associated with PPCs (Table 5).

\section{Discussion}

In this study, we examined the effectiveness of short-term cessation for preventing the development of PPCs. We instructed active smokers to quit smoking when they were scheduled for surgery. The patients remained off cigarettes for at least 4 weeks prior to surgery in order to reduce the incidence of PPCs. The patients who could not stop smoking before surgery, surgery was postponed until they managed to discontinue smoking for 4 weeks. Patients who continued to smoke until surgery were not included in this study; thus, we could not compare the current smokers with patients who continued short-term cessation. According to the previous reports, the frequency of PPCs in current smokers who underwent pulmonary resection ranged from $23 \%$ to $43.2 \%$ (15). Fifteen percent of the 
Table 4 The Incidence of PPCs according to smoking status

\begin{tabular}{|c|c|c|c|c|}
\hline Variable & Never smoker $(n=287)$ & Former smoker $(\mathrm{n}=361)$ & Abstainer $(n=105)$ & All cases $(n=753)$ \\
\hline Atelectasis & 0 & $1(0.3 \%)$ & $3(2.9 \%)$ & $4(0.5 \%)$ \\
\hline Bronchopleural fistula & 0 & 0 & 0 & 0 \\
\hline Chylothorax & $2(0.7 \%)$ & $1(0.3 \%)$ & 0 & $3(0.4 \%)$ \\
\hline $\begin{array}{l}\text { Acute exacerbation of } \\
\text { Interstitial pneumonia }\end{array}$ & 0 & $2(0.6 \%)$ & $2(1.9 \%)$ & $4(0.5 \%)$ \\
\hline Pneumonia & $4(1.4 \%)$ & $11(3.0 \%)$ & $6(5.7 \%)$ & $21(2.8 \%)$ \\
\hline Prolonged air leakage & $11(3.8 \%)$ & $9(2.5 \%)$ & $6(5.7 \%)$ & $26(3.5 \%)$ \\
\hline
\end{tabular}

PPCs, postoperative pulmonary complications.

Table 5 Univariate and multivariate analyses to identify predictors of PPCs

\begin{tabular}{|c|c|c|c|c|}
\hline Variable & \multicolumn{2}{|c|}{ Univariate } & \multicolumn{2}{|c|}{ Multivariate } \\
\hline \multicolumn{5}{|l|}{ Age } \\
\hline $75 \geq$ & $1.784(0.945-3.673)$ & 0.076 & & \\
\hline $75<$ & $1.0(-)$ & & & \\
\hline Male & $2.154(1.231-3.938)$ & 0.007 & 1.949 (1.023-3.832) & 0.043 \\
\hline Female & $1.0(-)$ & & & \\
\hline \multicolumn{5}{|l|}{ Performance status } \\
\hline 1,2 & $1.472(0.588-3.192)$ & 0.382 & & \\
\hline Abstainer & 2.353 (1.245-4.255) & 0.010 & $1.799(0.906-3.457)$ & 0.095 \\
\hline Never and former smoker & $1.0(-)$ & & & \\
\hline \multicolumn{5}{|l|}{ Pack year smoking } \\
\hline $20 \geq$ & 1.932 (1.079-3.144) & 0.025 & $1.178(0.621-2.264)$ & 0.619 \\
\hline $20<$ & $1.0(-)$ & & & \\
\hline \multicolumn{5}{|l|}{ Surgical procedure } \\
\hline Standard resection & 3.643 (1.670-9.577) & $<0.001$ & 3.368 (1.414-9.677) & 0.005 \\
\hline Sublobar resection & $1.0(-)$ & & & \\
\hline
\end{tabular}

PPCs, postoperative pulmonary complications; $\mathrm{Cl}$, confidence interval; HR, hazard ratio; standard resection: lobectomy and pneumonectomy; sublobar resection: segmentectomy and wedge resection. 
patients in the abstainer group in this study suffered from PPCs, including atelectasis, exacerbations of interstitial pneumonia, pneumonia, and prolonged air leakage. Shortterm cessation might have some effect on the prevention of PPCs after pulmonary resection.

On the other hand, the patients in the short-term cessation group experienced more PPCs in comparison to the other groups. In addition, cigarette smoking had a harmful effect on the short-term post-operative outcomes. The abstainer group required tube drainage for a longer period and longer hospitalization in comparison to the former smoker and never smoker groups. According to these results, a longer smoking cessation period might be more effective for reducing the risk of PPCs. Recently, Fukui et al. reported the effectiveness of smoking cessation on surgical outcomes in patients with primary lung cancer (16). They divided the patients in detail according to the duration of smoking cessation and examined the relationship between the preoperative interval of smoking cessation and PPCs after surgery. They concluded that a longer period of cessation might be more effective for reducing the risk of PPCs (16). On the other hand, treatment delays may cause tumor progression; thus, we should not extend the smoking cessation period without reason. Samson et al. reported that patients receiving delayed resection for clinical stage I NSCLC had higher comorbidity scores, which might have affected the ability to perform lobectomy and resulted in higher perioperative mortality (17). They also reported that delaying surgery was independently associated with increased rates of upstaging and decreased median survival in clinical stage I NSCLC (17). Maiga et al. also reported the interval of time between the identification of primary lung cancer and resection (18). They reported that the median time from the identification of a tumor to resection was 98 days (range, 66-139 days), and no correlation was observed between tumor progression and the interval of time (18). If we provide antismoking education in parallel with the identification of the tumor, the adverse effects associated with treatment delay may be diminished.

Perioperative smoking cessation has various favorable effects besides reducing PPCs (19,20). A smoking cessation program initiated before surgical lung resection was reported to be cost-effective at both 1 and 5 years postsurgery (19). In comparison to never smokers, highly nicotine-dependent male patients who were deprived of cigarettes experienced more severe pain after thoracoscopic radical lung cancer surgery (20). Moreover, preoperative smoking cessation at least 3 weeks before surgery led to better postoperative pain outcomes than smoking cessation within 3 weeks of surgery (20). Balduyck et al. reported that smoking cessation was beneficial at any time point prior to lung cancer surgery and that current smoking at the time of surgery was associated with a poor postoperative quality of life (21).

The present study was associated with several limitations, including its retrospective observational study design. This study used a multi-institutional Japanese database and only enrolled patients who had undergone surgical resection. A prospective study may have been more appropriate for assessing the effects of smoking cessation guidance. On the other hand, few reports have evaluated the effectiveness of smoking cessation guidance at multiple facilities. Another limitation of this study is definition of the smoking status. We the used self-reported smoking cessation period in this study; thus, we cannot deny the possibility that some patients secretly smoked during the cessation period. Actually, Sivasithamparam et al. examined the biochemical confirmation of cigarette use via saliva samples for the determination of the long-acting nicotine metabolite cotinine (22). A cotinine analysis indicated that one of four patients who denied cigarette use in the past month misrepresented their true smoking status. They concluded that self-reported measures might not accurately depict the true smoking status (22). We should evaluate the effects of smoking cessation with the measurement of metabolic products of nicotine in a further study.

In conclusion, although abstaining from smoking for at least 4 weeks prior to surgery was not enough to reduce the incidence of PPCs, all patients should be advised and counseled to quit smoking as soon as possible before lung resection.

\section{Acknowledgments}

We thank Japan Medical Communications (https://www. japan-mc. co. jp/) for editing drafts of this manuscript. Funding: None.

\section{Footnote}

Reporting Checklist: The authors have completed the STROBE reporting checklist. Available at http://dx. doi. org/10. 21037/jtd-20-2574

Data Sharing Statement: Available at http://dx.doi. 
org/10.21037/jtd-20-2574

Peer Review File: Available at http://dx. doi. org/10.21037/ jtd-20-2574

Conflicts of Interest: All authors have completed the ICMJE uniform disclosure form (available at http://dx. doi. org/10.21037/jtd-20-2574). The authors have no conflicts of interest to declare.

Ethical Statement: The authors are accountable for all aspects of the work in ensuring that questions related to the accuracy or integrity of any part of the work are appropriately investigated and resolved. The study conformed to the provisions of the Declaration of Helsinki (as revised in 2013). The Institutional Review Board at the Hiroshima Red Cross Hospital and Atomic Bomb Survivors Hospital (IRB number: H30-672) and informed consent was obtained from the patients.

Open Access Statement: This is an Open Access article distributed in accordance with the Creative Commons Attribution-NonCommercial-NoDerivs 4.0 International License (CC BY-NC-ND 4.0), which permits the noncommercial replication and distribution of the article with the strict proviso that no changes or edits are made and the original work is properly cited (including links to both the formal publication through the relevant DOI and the license). See: https://creativecommons.org/licenses/by-nc-nd/4.0/.

\section{References}

1. Creamer MR, Wang TW, Babb S, et al. Tobacco Product Use and Cessation Indicators Among Adults United States, 2018. MMWR Morb Mortal Wkly Rep 2019;68;1013-9.

2. Health at a Glance 2015 OECD Indicators. Available online: https://doi.org/10.1787/health_glance-2015-en (June 8 2020, accessed).

3. Chen W, Thomas J, Sadatsafavi M, et al. Risk of cardiovascular comorbidity in patients with chronic obstructive pulmonary disease: a systematic review and meta-analysis. Lancet Respir Med 2015;3:631-9.

4. Alberg AJ, Brock MV, Ford JG, et al. Epidemiology of lung cancer: Diagnosis and management of lung cancer, 3rd ed: American College of Chest Physicians evidence-based clinical practice guidelines. Chest 2013;143:e1S-e29S.
5. Ordóñez-Mena JM, Schöttker B, Mons U, et al. Quantification of the smoking-associated cancer risk with rate advancement periods: meta-analysis of individual participant data from cohorts of the CHANCES consortium. BMC Med 2016;14:62.

6. Mehta LS, Watson KE, Barac A, et al. Cardiovascular Disease and Breast Cancer: Where These Entities Intersect: A Scientific Statement From the American Heart Association. Circulation 2018:137:e30-e66.

7. Parsons A, Daley A, Begh R, et al. Influence of Smoking Cessation. After Diagnosis of Early Stage Lung Cancer on Prognosis: Systematic Review of Observational Studies With Meta-Analysis. BMJ 2010;340:b5569.

8. Okamoto T, Suzuki Y, Fujishita T, et al. The prognostic impact of the amount of tobacco smoking in non-small cell lung cancer-differences between adenocarcinoma and squamous cell carcinoma. Lung Cancer 2014;85:125-30.

9. Nakagawa M, Tanaka H, Tsukuma H, et al. Relationship between the duration of the preoperative smoke-free period and the incidence of postoperative pulmonary complications after pulmonary surgery. Chest 2001;120:705-10.

10. Barrera R, Shi W, Amar D, et al. Smoking and timing of cessation: impact on pulmonary complications after thoracotomy. Chest 2005;127:1977-83.

11. Mason DP, Subramanian S, Nowicki ER, et al. Impact of smoking cessation before resection of lung cancer: a Society of Thoracic Surgeons General Thoracic Surgery Database study. Ann Thorac Surg 2009;88:362-70.

12. Rodriguez M, Gomez-Hernandez MT, Novoa N, et al. Refraining from smoking shortly before lobectomy has no influence on the risk of pulmonary complications: a casecontrol study on a matched population. Eur J Cardiothorac Surg 2017;51:498-503.

13. Batchelor TJP, Rasburn NJ, Abdelnour-Berchtold E, et al. Guidelines for enhanced recovery after lung surgery: recommendations of the Enhanced Recovery After Surgery (ERAS $\left.{ }^{\circledR}\right)$ Society and the European Society of Thoracic Surgeons (ESTS). Eur J Cardiothorac Surg 2019;55:91-115.

14. Katayama H, Kurokawa Y, Nakamura K, et al. Extended Clavien-Dindo classification of surgical complications: Japan Clinical Oncology Group postoperative complications criteria. Surg Today. 2016;46:668-85.

15. Zaman M, Bilal H, Mahmood S, et al. Does getting smokers to stop smoking before lung resections reduce their risk? Interact Cardiovasc Thorac Surg 2012;14:320-3. 
16. Fukui M, Suzuki K, Matsunaga T, et al. Importance of Smoking Cessation on Surgical Outcome in Primary Lung Cancer. Ann Thorac Surg 2019;107:1005-9.

17. Maiga AW, Deppen SA, Pinkerman R, et al. Timeliness of Care and Lung Cancer Tumor-Stage Progression: How Long Can We Wait? Ann Thorac Surg 2017;104:1791-7.

18. Samson P, Patel A, Garrett T, et al. Effects of delayed surgical resection on short-term and long-term outcomes in clinical stage I non-small cell lung cancer. Ann Thorac Surg 2015;99:1906-12.

19. Slatore CG, Au DH, Hollingworth W. Costeffectiveness of a smoking cessation program

Cite this article as: Takenaka T, Shoji F, Tagawa T, Kinoshita F, Haratake N, Edagawa M, Yamazaki K, Takenoyama M, Takeo S, Mori M. Does short-term cessation of smoking before lung resections reduce the risk of complications? J Thorac Dis 2020;12(12):7127-7134. doi: 10.21037/jtd-20-2574 implemented at the time of surgery for lung cancer. J Thorac Oncol 2009;4:499-504.

20. Zhao S, Chen F, Wang D, et al. Effect of preoperative smoking cessation on postoperative pain outcomes in elderly patients with high nicotine dependence. Medicine (Baltimore) 2019;98:e14209.

21. Balduyck B, Sardari Nia P, Cogen A, et al. The effect of smoking cessation on quality of life after lung cancer surgery. Eur J Cardiothorac Surg 2011;40:1432-7.

22. Sivasithamparam J, Visk CA, Cohen EE, et al. Modifiable risk behaviors in patients with head and neck cancer. Cancer 2013;119:2419-26. 